\title{
Ethical Placements? Under What Conditions Can Educational Placements Support Sustainable Development?
}

\section{INTRODUCTION}

Understanding the impact of human mobility on various forms of knowledge transfer and economic development has formed the subject of recent debate. Our sister book (Ackers and Ackers-Johnson 2016) is devoted to a wider discussion of the impact of the deployment of professional (i.e. qualified) volunteers on the Ugandan public health system. If there is a dearth of research on this topic and some scepticism about the impact of very highly skilled professionals undertaking extended stays in low resource settings, then it is fair to say that our understanding of the impact of short stay undergraduate electives is more or less non-existent. The literature on volunteer-tourism or 'voluntourism' has begun to raise uncomfortable questions but mainly within the frame of 'gap year' sojourns (Simpson 2004; Snee 2013). As we have noted, to the extent that there is any published research on electives it is almost exclusively focused on medical students (Coates 2006; Drain et al. 2007; Rominski et al. 2015). Some of this literature, whilst primarily focused on the (clinical) gains to the students themselves and the risks of what are quite often self-organised individual ventures, has hinted at the potential burden placed upon host locations (BMA 2009; Rominksi et al. 2015). As with all forms of 'volunteering' there is a presumption of benevolence, of altruism and inherent good or at least neutrality; of 'no harm'. But peppered within this fluffy 
celebratory glow is a thorny question: in a context when the UK is itself struggling to place, train and fund its own, why should we expect low resource settings to take responsibility for training its future health workforce?

Our role in the deployment of long-term volunteers has made us very aware of the need for careful recruitment and deployment within structured, supported and managed programmes if we are to avoid becoming part of the problem and undermining health systems. Indeed, this is an unfolding story of 'unintended consequences.' The EEP model builds on eight years' experience of managing volunteers in the context of the Ugandan public health system. Unlike other elective placement schemes run by private companies keen to profit from the burgeoning demand for international placements, the EEP model grew out of our Sustainable Volunteering Programme and is firmly situated within an active and evidence-based partnership-based engagement. The ethics and sustainability of our engagement has formed a key component of project design and evaluation.

Building on our previous (exploratory) experience of deploying medical students alongside professional volunteers we anticipated two key dimensions of (positive and sustainable) impact. In the first instance, we knew that professional volunteers working for long periods in highly stressful circumstances and often with reluctant learners and absent colleagues were often 'refreshed' and re-energised when UK students arrived. Secondly, having witnessed some very bad practice involving payments for supervision by small poorly organised income-generating electives programmes, we were aware of the pitfalls of cash inducements to local supervisors/ mentors. These payments typically failed to guarantee any supervision on the ground and contributed to systemic corruption. On the other hand, we witnessed the important contribution even quite small strategic investments could make to removing simple 'snags' that debilitate local health systems. Mdee and Emmott (2008) define the 'tension between operating a viable and commercially-sustainable enterprise and maximising social and development impacts' in the context of what they call 'pro poor tourism' (p. 191). Whilst we would distinguish undergraduate electives from gap year tourism the tension remains and lies at the heart of the EEP mission. The authors advocate the extension of a certification process based on Fair Trade Principles to kitemark organisations involved in volunteer deployment. Informed by these principles, the EEP project sought to design a model that reduced the risks of contributing to global 
inequality and corruption (through the commodification of placements) or systems dependency (on consumables provision for example) and enabled us to make systems enhancing investments.

Perhaps influenced by the somewhat negative experiences of 'free mover' medical electives ${ }^{1}$ compounded by the voluntourism debates we had rather anticipated students aiding the knowledge brokering functions of professional volunteers and seriously underestimated the role that students themselves, when appropriately placed, can play in knowledge exchange processes. Our research has evidenced the important role that students can play in actively supporting mutual learning on-the-job in health facilities but also in conjunction with undergraduate teaching programmes. And, linked to this peer mentoring role, we have found that the student placements have had a significant motivational impact on health workers in the facilities they have been based in. Finally, as the project has developed we have sought to incentivise and invest in the skills and experiences of local health workers. This is not to suggest that Ugandan health workers lack the skills and experience to supervise UK students. But we are very aware of marked differences in role delineation, training and experience. With that in mind we have encouraged bi-lateral professional exchanges supporting Ugandan health workers to apply for fellowships to spend time in the UK. With the support of British Commonwealth Professional Fellowships, we have been able to bring a number of colleagues over from both the University and the Health District to experience British professional education and placement schemes and intensify the quality of relationships.

We have noted elsewhere the externality effects associated with 'gapfilling' and labour-substitution roles. Deploying volunteers in service delivery undermines local health systems (Ackers and Ackers-Johnson 2016). It is also associated with high levels of personal risk for volunteers. This is heightened in the case of students. As a result, all of our engagement is underpinned by the 'co-presence' principle reducing to a minimum the incidence of lone working. Our objectives are system-focused and we hope that such a focus will in the medium term improve the care of all patients and not just those whose lives we personally touch. Having said that we are acutely aware that very many patients have benefited on a daily basis from their encounters with

${ }^{1}$ Opengart uses the concept of 'free agent learners' in the context of boundaryless careers. In the past many medical students negotiated their own placements as individual 'pioneers'. 
our students. Patients comment regularly on the care and compassion shown to them by British professionals and students and we are in absolutely no doubt that our students have saved the lives of mothers and babies and improved the quality of lives for many others.

Students were invited, in the survey, to comment on the impact they believed their placement had on the individuals, facilities, organisations and health system in the country within which they were placed. This is obviously a very broad and subjective question, and the students' responses may not be indicative of their actual impact. However, responses were very positive. Fifty-four percent of students believed they had a very positive impact, $34 \%$ a slightly positive impact and $9 \%$ no impact. Three percent of students were unsure, however no students believed their placement had a negative impact. The responses for students travelling to Uganda were generally more positive than those travelling to India, which is likely to be related to the fact that the India placement were observational only whereas the Uganda placements allowed for supervised hands-on practice as mentioned previously. Additionally, the health facilities and staffing in India were more advanced than those in Uganda which left more scope for students to try to make improvements in the Ugandan health facilities.

The remainder of this chapter assesses the ethics of placing students in Uganda because, as we have explained, the placements in India were organised on a very different basis. We had no prior engagement in India and the placements were organised by other parties in a private, not-forprofit health facility. The entire programme was managed by the Indian organisation which charged a fee for every student of $£ 850$. $^{2}$ It was clear from the start that these were observation only placements and students were prohibited from any involvement with patients or wards and they included a two-week non-clinical cultural exposure designed specifically for these and many other foreign students. The India placements did not set out to support local capacity-building or systems change and took place largely outside the Indian public health system. As such we do not regard these as falling within the model of 'Ethical Educational Placements'.

Chapter 2 emphasised the importance of systems change and capacitybuilding to all of our work in Uganda. This implies a focus on public

${ }^{2}$ This is usual practice with companies active in the electives market. What distinguishes this placement, however, is that there was no intermediary organisation in the UK taking a slice of the profits. 
health systems and we have questioned the ethics and sustainability of investing in parallel service development in the private and not-for-profit sectors. ${ }^{3}$ In this context, the chapter examines first the 'knowledge premium' associated with placements asking whether students play a role in the transfer and exchange of useful knowledge and skills. It then moves on to discuss the role that the EEPs have played in creating new opportunities for the kinds of active knowledge mobilisation necessary to bring about sustainable systems change.

\section{Supporting Professional Volunteer Engagement AND EFFICACY}

Our decision to embark on the development of an ethical placements programme stemmed directly from our role in managing and evaluating the Sustainable Volunteering Program (SVP). The SVP deployed longterm highly skilled professional volunteers within the frame of the Ugandan Maternal and Newborn Hub to promote sustainable improvements in public health systems (as outlined in Chapter 2 and in Ackers and Ackers-Johnson 2016). Understanding the contribution that even these qualified volunteers can make towards sustainable system change has proved a real challenge. What we have learnt, over a period of many years, is that volunteer deployment and management is critical to mitigation of potential systems damage. Linked to this we have observed the mutual benefits of using long-term volunteers as anchors for short stays (Ackers 2015); short stays in the absence of continued project and personnel presence on the ground are rarely successful and can lapse into damaging forms of service delivery. ${ }^{4}$ On the other hand, when there is continuity of clearly defined projects with on-going bridging relationships, short-term stays can be highly beneficial both in terms of harnessing critical skills and motivational impacts. And, having long term volunteers on the ground preparing for and creating the 'sticky branches' (Meyer 2003) that lubricate short stays enables short stay professionals to hit the

${ }^{3}$ Case Study 5 outlines work undertaken by a placement group focused on the development of a Public-Private-Partnership as outlined in the Ugandan Ministry of Health's Strategic Plan, 2012.

${ }^{4}$ It is continued presence over time rather than the length of individual stays that is of importance here. 
ground running' and become effective knowledge brokers almost immediately. In this case, short stayers are most commonly very senior professionals unable to commit to longer periods away from work. However, we observed similar effects with short-stay medical electives; the Professional Volunteers were often excited to have students join them to support their own plans for audits or specific interventions. Motivation over the course of long-term placements often wanes in the face of on-going challenges (Gedde et al. 2011). At times, local health workers appear disinterested in training and long-term volunteers gain motivation from having knowledgehungry UK students on the wards as the following volunteer suggests:

They (group of nursing students) have been some of the best students I've ever supervised. They were always on time, keen to learn and got straight on with whatever needed to be done that day... It removes some of the stress having more people around to help out. (PV, Uganda)

The SVP came to an abrupt end, as with many development interventions, when our knowledge of how to mobilise and optimally place volunteers was at its peak. This is an on-going problem with Aid with many projects; jumping between funded programmes and defining themselves according to the demands of funders rather than the evidence-base and longer term strategic but iterative objectives. We realised that if we were able to mobilise an effective placement programme and generate an overhead that could fund the placement of long-term volunteers, we could achieve the kind of symbiotic sustainability capable of achieving incremental evidence-based systems impact.

Chapter 3 has discussed the benefits of long term volunteers to student support and learning. Our concern in this chapter is to explain how the model as a whole underwrites the deployment of long-term volunteers (as an outcome for host institutions and systems). It could be argued that investing in mobile expertise from the UK (in the form of professional volunteers) is in itself an example of neo-colonialism. Why not utilise local expertise to fulfil the supervisory role? Sadly, our experience of managing PVs in Uganda has exposed the fundamental weaknesses of human resource management systems in the Ugandan public health system. Elsewhere, we have argued that the failure to implement effective human resource management processes to counter endemic absenteeism and poor time-keeping lies at the heart of maternal morbidity and mortality (Ackers et al. 2016). Absenteeism is a major problem in Uganda and many staff either fail to come to work at all or, 
when they do, tend to show very poor attitudes towards time-keeping. This problem is common to many low resource settings where systems of accountability are not in place and creates a specific challenge for the organisation of undergraduate placements. This is one of the key reasons why professional volunteers are so important to minimise the risks associated with lone working and ensure effective supervision in often extremely stressful circumstances. Asked whether the impact of the EEP would be the same without the presence of professional volunteers, a lecturer at Mountains of the Moon University replied:

If you don't they (the UK students) won't get supervised - it would be dangerous to do that.

In a far more complex process, the deployment of professional volunteers in turn supports the development of training clusters that have a centrifugal effect in drawing in local health workers and students. Put more metaphorically; the professional volunteers can be characterised as the eyes of creative (and disruptive) knowledge mobilisation storms. But building and sustaining clusters is labour intensive. It requires organisation, planning, active bi-lateral communication and on-the-ground copresence. These clusters and the benefits accruing from them cannot be achieved by simply posting large volumes of students in low resource settings and paying a cash premium. The following respondent (a member of staff at the local university) responds to the question, 'Could we have MMU and our students working alongside on placement?'

Yes, that would be great so long as we don't have too many there at once - it depends a bit on the timing and numbers. It would be good if we communicated a bit better and tried to line it up a bit more.

As we have noted above, during the SVP it was rare to see anything other than medical students on electives. The opportunity to deploy much wider cadres of students has contributed significantly to this process creating opportunities for multi-disciplinary exchanges supporting the work of professional volunteers in a more holistic way. An example of this can be seen in the deployment of child nursing students into the neonatal units linked to maternal facilities. This has provided critical support to our obstetric and midwifery volunteers attempting to care for mothers and babies from pregnancy until they are able to go home. It provided 
opportunities for students to 'spoke out' to witness birthing processes and immediate neonatal care, and then follow this through to the neonatal unit. In effect, it enables the volunteers to be in two places at once but always at hand if the students need support. In the process the UK nurses are able to work alongside, and share their skills and experience with, local staff and students. In a similar vein, students have been able to be actively involved in ante-natal or baby clinics, in laboratory-based testing of patients or in record keeping and management, all of which support the complex interventions of long-term volunteers. It is clear from our research that undergraduate students play an active role in augmenting the knowledge brokerage function of long-term volunteers.

\section{Students as Knowledge Brokers}

Having conceptualised UK professional volunteers as knowledge brokers (Ackers and Ackers-Johnson 2016) we are nevertheless aware of some tension between the needs and ability to exercise long-term mobility on the part of UK professionals, on the one hand, and the expressed needs of host institutions for the most senior clinicians. Our experience of the volunteers has challenged received wisdom on the part of UK professional bodies (such as the Royal Colleges), volunteer deployment agencies (such as VSO) and Ugandan hosts that only very senior clinicians are suitable and able to make a serious contribution. Indeed, some of the most effective volunteers have been more junior doctors willing to engage in some of the more mundane back-to-basics systems repair work. We were not expecting students, caricatured in roles as learners (inert sponges) rather than 'teachers' to assume the role of knowledge brokers. Our research indicates quite the opposite; that the students we have selected, in the positions we have placed them and with the support in place, have played very active and meaningful knowledge brokerage roles. Many interventions (such as triage, infection control or medical records audit for example) benefit from having more human resource to assist. The emphasis on 'back-to-basics' or 'neglected processes' ${ }^{5}$ in global health lends itself well to less experienced cadres especially when they are carefully

${ }^{5}$ The concept of 'neglected processes' was utilised within the frame of the Ugandan Maternal and Newborn Hub interventions at the suggestion of Dr Simon Mardel. 
supervised and managed. Mardel, a leading expert of ebola-preparedness work, describes the key global health challenges as follows:

The greatest currently achievable gains in global healthcare appear dependent on solving the currently 'neglected' processes in organisation and delivery of health care and health education. These deficits, aimed at disease prevention, early diagnosis, self-care, low cost intervention, early recognition of severity, appropriate and safe use of existing resources are not sufficiently covered by existing specialties, or their redress requires unprecedented collaboration. ${ }^{6}$

We have reproduced Mardel's '7 pillars' in Fig. 4.1 to illustrate the potential contribution that undergraduate students can make to global health. Improving these basic processes such as patient monitoring, record keeping, IPC and access to information are precisely the areas where students have the knowledge and skills to engage effectively with local health workers and health systems in critical public and preventive health roles. This explains why, in many respects, we believe that knowledge mobilisation clusters combining undergraduate students with UK midwifery, nursing and AHP volunteers (and their Ugandan peers) deliver optimal impact.

One of the contexts within which this knowledge brokerage role has occurred most proactively has been through their engagement with Ugandan students. We had anticipated and planned to 'buddy' the students with our partner University (Mountains of the Moon) and this has created opportunities for more formal mutual learning encounters; similar to the buddy system employed in India. Many of the students have taken advantage of this opportunity and planned joint workshops and seminars with their student peers at MMU and Makerere University. One of the local supervisors made the following comment on peer learning showing its motivational impact on them as trainers and the students involved:

It was a great opportunity to sit together and analyse; 'what do you study that we don't study and what do we study that you don't study' so it was a great opportunity for all the students. And to us, the trainers, it was very interesting to know how students from the UK think and how they look at things because that guides us on what we can also tell our students. You

\footnotetext{
${ }^{6}$ Cited at http://www.nhsevents.org/img/events/213/Dr\%20Simon\% 20Mardell.pdf
} 




Fig. 4.1 The seven pillars of neglect in global health. Source: Acknowledgement to come

know we are always limited with some few things and sometimes we pick-up the perception of maybe we cannot get this far but with the interaction we had with them we realised that yes if we pick up that kind of thinking and try to initiate it into our students then we'll have the best out of this program.

This programme is continuing to evolve and we are currently hosting this respondent in the UK on a 5-month Commonwealth Professional Fellowship focused on prosthetics and robotics. The Fellowship is designed to build relationships for future undergraduate placements but 
also to support capacity-building in Uganda in the development of prosthetics. This in turn represents an immediate response to a very tragic case of domestic violence (Case Study 1):

\section{Case Study 1: Holistic Responses to Domestic Violence}

In June 2016, a young mother from a very isolated rural area was brutally attacked by her partner who hacked off both of her hands and caused serious injury to her face including the loss of an ear and an eye. Ninsiima is a peasant farmer with two small children and was thirty-six weeks pregnant at the time of the attack. She was cared for, from an obstetric perspective, by a $\mathrm{K} 4 \mathrm{C}$ professional volunteer and gave birth safely. Students on the EEP placements then supported her to care for her baby in the newly set up neo-natal unit prior to her return home. At this point a group of young pioneering engineers that had been trained by the University of Salford/K4C biomedical engineering project ${ }^{7}$ under the leadership of Dr Ssekitoleko responded to the situation. One of these engineers ( $\mathrm{Mr}$ Senabulya) became involved in assessing the possibility of building prosthetic hands for Ninsiima. This lead to a successful application for funding to the British Commonwealth Fellowship programme, enabling Mr Senabulya to come to Salford University for five months to work with colleagues in robotics and prosthetics. The objective of this fellowship is to build capacity in Uganda in the production of prosthetics for low resource settings, assess the possibility of building two hands for Ninsiima whilst also working alongside the UK placement team to build on our successful prosthetics and orthotics placements. One of the EEP students placed in this environment explains her experience:

The first two weeks were based in the largest referral hospital in Uganda. We were looking at things like fractures, spinal injuries and club foot. We were helping out as much as we could and seeing patients on a daily basis and looking at the challenges they faced and trying to sort out solutions to make it easier for them by reducing the cost of things and the kind of materials they needed because the lack of resources was one of the main problems over there. The second two weeks we were with the students from the orthopaedic medical school, essentially it is just the same course as we are doing. They

${ }^{7}$ This project is funded by the Tropical Health and Education Trust. For details see http://www.salford.ac.uk/research/care/research-groups/knowledge-and-place 
were doing prosthetics, dealing with wheelchairs and crutches. We were seeing what we could learn from them and teach them what we have learned here. (Prosthetics \& Orthotics, Uganda)

EEP students have also provided considerable support to our PVs involved in the assessment of the MMU midwifery degree students in OSCI-type clinical scenarios. ${ }^{8}$ All of the people involved in these have found them immensely valuable. One of the MMU lecturers expresses her views about the students' contribution:

Interviewer: "Do you feel the students make an active contribution to Uganda - do they have skills to give or are they too junior?"

$M M U$ Lecturer: "I have met a few groups who have done some teaching with our students then on Saturday [the volunteer] had them come and help with a mock OSCI with the MMU students and I observed that. It's also helpful to our students in lectures - they see a student with a different capacity with more knowledge and skills.

Interviewer: "So you feel the UK students have more knowledge and skills than MMU students?"

MMU Lecturer: "Probably like with the clinical examinations [the volunteer] is using the UK students for the actual exam; they can read an exam question and act appropriately; they know the practical side. The MMU students don't. Observing the UK students on the wards in comparison to other nursing schools in Fort Portal who send students with zero clinical skills; your students are teaching these Ugandan students on the wards. Our MMU midwives are pretty experienced but many of the other students in Fort Portal are not. The [EEP students'] clinical practice is different and at a higher standard than here so they are model clinicians in a student role. [LTV] has commented that she really thinks they add a lot to the general student population who come to the hospital for clinical placements. And with the SVP volunteers mentoring your students there is a wealth of knowledge - a real cluster which is of real benefit to the local students.

${ }^{8}$ An 'objective structured clinical examination' (OSCE) is a modern type of 'hands-on' examination often used in health sciences designed to test clinical skill performance and competence. (https://en.wikipedia.org/wiki/Objective_ structured_clinical_examination) 
EEP students have also been actively engaged in CPD-style training in health facilities and the community. Nursing and paramedic EEP students developed bespoke first aid training programmes for cadres of staff who have never had the opportunity for training; most notably these included all the local ambulance drivers and a group of potential paramedics. Wherever possible, we support opportunities for training interventions involving the students. As a project, we have a firm policy on cash per diems (we never pay them) and we endeavour to hold the training as close as possible and where we can within the clinical environment to reduce time out of work (see Ackers and Ackers-Johnson 2016). What we had perhaps underestimated was the very important knowledge brokerage role that EEP students played in relation to other (Ugandan) students and health workers. Here the benefits were mutual with active exchanges of skills and experience taking place. In the case of many Ugandan students, ${ }^{9}$ the more advanced knowledge/experience of the UK students (most of whom are at the end of their second year and/or mature students) meant that the UK students filled the roles of the usually absent local supervisors.

We noted above the involvement of UK students in paramedic training to ambulance drivers and nurses. At first we had some anxiety about placing students from Allied Health Professions that do not (yet) exist in Ugandan public services. These concerned, in particular, paramedics and podiatrists. It proved difficult to explain to many Ugandan health workers what a podiatrist was. However, we were able to place them in a club foot and diabetic clinic in a local hospital and this precipitated a strong demand for more such students on the part of the hospital and the expression of interest in further developing work on diabetic foot ulcers (a speciality of some of the Salford tutors). Similarly, discussions with the District Health Officer about the placement of paramedics raised the possibility of working towards a policy of requiring a local health worker to accompany patients on ambulance journeys alongside the drivers (who currently travel

\footnotetext{
${ }^{9}$ We are referring here to the large numbers of new entrants into certificate and diploma level nursing studying at colleges in Fort Portal and not to the Diploma qualified experienced midwives on the MMU degree.
} 
unaccompanied). How far ideas such as they progress remains to be seen; what we can say with some certainty is that the placement of students and exposure to the diverse professions has had an impact in terms of policy transfer. As a project, we make careful decisions about whether and how to invest in these project ideas that arise during placements in a very cautious and iterative fashion.

In the next case the manager of one of our partner organisations, an experienced British physiotherapist, explains how she felt the students contributed to her own reflective practice and to the mentoring of Ugandan staff within the organisation:

It was really good for us to learn from them because they kept on asking why we do things - it makes you as a clinician think about what you are doing more. Instead of just doing what I do I have to explain it and think about it. It's what Ugandan students don't do. They're anxious about questioning. Our physios here enjoy learning from the UK students. They bring the latest research; local staff don't have the same level of education.

The point about local staff reflects in many ways the nascence of some of these allied health professions in low resource settings such as Uganda. It is not a comment about the innate abilities of local staff or students but the fact that most of them will be qualified at certificate or diploma level. In comparison, many of the UK physiotherapy students already held a first degree. The respondent (above) goes on to make a point about length of stay in the placement setting suggesting that from the perspective of her investment and the benefits of the local organisation students are best placed in one location for most of the time:

We try to do a lot of teaching and set objectives for each student. Throughout their first week we looked at their strengths and weaknesses, and say ok let's work on your weaknesses while you are here! So, it's very individualised and that's what we can do when somebody is here for 3 weeks, but not when somebody is only here for 2 days. And also, you don't want to put so much time and effort in, I just don't have the same interest because in 2 days they're not going to learn that much from me, as opposed to three weeks, they have a huge amount of things they can learn, we can sit down and do teaching sessions and discuss cases. But 
I'm not going to do that with somebody who's only here for 2 days, because it's a lot of time and effort on my part and they don't get the same out of it. It's not fair on the parents, that you constantly have a new person working on their child.

Another Ugandan supervisor makes a similar comment suggesting he would prefer stays of two months:

Interviewer:
use to Uganda - are they more of a burden than a
benefit?
Ugandan Supervisor: "No, I wouldn't call it a burden because learning is
both sides; some things we learn from them so it is
experience sharing. It isn't a burden the only challenge
is the time they spend - if it is not too much time then
the orientation ends when their period here is ending.
In one month they get used to everything and then the
second month they could perform but we do benefit".

This is an interesting issue which reflects some creative tension between the wishes of students, in some cases, to experience a breadth of settings and exposures. The EEP placement team have tried to balance these needs and explain to the students the risks of volunteer tourism and voyeurism and the importance to their learning and impacts of relationship-building. Rominksi et al refer to this as a necessary process of 'expectation management' (2015: $3)$. On the other hand, some students found their placements extremely demanding. This very much depended on the context rather than the student in question. Placements on a very busy neonatal unit whilst very interesting and rewarding (and the envy of many EEP students) are exhausting. In this situation one of the students felt that a day a week in another setting was helpful:

It was nice to have one day a week to do something that gave relief and fun.

Something through which you could understand the community a bit more.

Like the health clinic, that was good. (Midwife, Uganda)

The compromise we have developed is to give students a flexible half day on a Friday to enable them to negotiate visits to other settings whilst 
keeping them firmly anchored in their primary location. This process in itself forms a critical part of student learning about global health.

\section{The 'Fair Trade Premium': Local Investment Without Fostering Corruption}

One of the most obvious ways to compensate or reimburse hosts in low resource settings for the work they do in supervising and mentoring UK students is via direct cash payments and, arguably, this is the mechanism that extends optimal autonomy to partners in the host setting enabling them to identify their own investment objectives. Many for-profit placement providers make cash payments directly into organisations (hospitals) as is the case in the Makerere/Mulago International Electives Programme or, often more informally, to local in-charges or senior health staff on the wards. In our experience neither of these approaches avoids the pitfalls associated with endemic corruption especially in the public sector or puts systems in place to guarantee supervision. Student placements become commodified and students are treated as 'cash cows' creating opportunities for personal gain. ${ }^{10}$

We have witnessed the 'institutional' approach in the National Referral Hospital where student placements have become an element of income generation, resulting not so much in a lack of supervision (in this incidence) but more in a kind of 'herding' of large volumes of students through wards in an almost voyeuristic enterprise. This process creates a serious burden for overwhelmed local staff and ward managers working in very congested facilities with few staff. One manager of a large maternity facility in Kampala told us that she felt that most of her time was spent supervising students. She was quick to point out that many of these were bussed into the hospital from local nursing and midwifery schools in large and unpredictable numbers but this was compounded by groups of visiting students from overseas. In addition to distracting local staff this herding process compounds physical congestion and existing problems of patient privacy, confidentiality and infection prevention control.

During the SVP, we also identified cases where small UK charities have engaged in student recruitment to aid their fund raising and organised, in

${ }^{10}$ Placements in the UK are remunerated on an inter-institutional basis but systems are in place to ensure accountability and supervision. 
good faith, at a distance cash payments to local clinical leaders. We have witnessed directly these payments being taken on a personal basis by senior (and mostly absent) managers with no funds trickling down to benefit the staff working on the ground. This creates tensions, jealousies and, most critically, results in lone working. We have discussed in some detail in our sister volume (Ackers and Ackers-Johnson 2016) issues around the commodification of training in Uganda and do not have space to rehearse these issues here. What is absolutely clear from all perspectives is that student placements have been recognised as a lucrative marketable commodity by both sending ${ }^{11}$ and receiving organisations resulting in some quite unethical outcomes.

In order to avoid these risks whilst also recognising the importance of some compensatory function we have adopted a 'Fair Trade Premium' approach. The previous section has evidenced the complexity of multilateral knowledge exchange processes associated with EEPs. Transferring knowledge in itself will fail to precipitate systems change in Ugandan public health facilities as knowledge mobilisation requires that health workers not only have knowledge but are also motivated to use that knowledge and have access to a basic modicum, of resource to facilitate behaviour change. ${ }^{12}$ In this context the 'Fair Trade Premium' is not simply compensatory; it represents a critical investment in knowledge mobilisation processes.

In practice this has involved setting aside $£ 150$ from each placement to support critical local investments that facilitate effective placements and systems improvement. The Premium takes the form of negotiated 'in-kind' investments: we never pay cash. Working with well-established local partners we identify 'snagging' problems that a small, immediate investment could overcome. Examples include the provision of a placenta pit in a facility where the lack of such a resource was the main factor preventing patient admissions. No amount of training or knowledge transfer could have got this maternity facility functioning if there was no way of disposing of placentas. We have also repaired or installed sinks as a precursor to hand hygiene training to support our infection

${ }^{11}$ It is certainly true to say that the clamouring gap year companies are making the biggest financial gains here not the hosting organisations.

${ }^{12}$ These relationships are discussed in more detail in Ackers and Ackers-Johnson (2016) (Chapter 4). 
prevention control interventions whilst also reducing risks for students. In other cases, we have constructed patient waiting areas to enable patients to wait under cover for out-patient treatment rather than congesting corridors. This also then created a visible space for students to set up a triage area and 'role model' the taking of basic observations and basic record-keeping. We have also undertaken some improvements to accommodation to enable doctors to remain on site during their duties; there was a property but it had fallen into some disrepair and needed some basic re-wiring and plumbing to connect water and power. Our intervention here was aimed at improving the medical presence to ensure $24 / 7$ cover as audit work had shown that the lack of medical presence was responsible for high levels of inappropriate referrals (and maternal and neonatal deaths). We also know that having doctors on site at all times created a better learning environment for our students. Other 'investments' included support to the university to provide transport for their own students (an on-going problem in Uganda) which would also in theory benefit the placement students and urgent repairs to an ambulance. We had some reservations about this last investment as we do not wish to get involved in covering any routine running costs or consumables. However, at the time none of the five ambulances were on the road and we wanted to utilise the opportunity of having three UK paramedic students to encourage the development of basic first-aid training to ambulance drivers and, more symbolically, to push policy makers to begin to consider the possibility of paramedic support to ambulance drivers. In practice, we were right to have such reservations as the ambulance was out of service again a month later. We have also purchased books for the university library and supported the costs of a mobile HIV/cancer screening/family planning clinic. ${ }^{13}$

The following section discusses these 'investments' in more detail to illustrate their knowledge mobilisation function. The following Ugandan ward manager describes her appreciation of the investments the EEP has made in the Regional Referral Hospital (RRH), the facility in which she was based:

${ }^{13}$ See Case Study 4 below. 
Interviewer:

Ward Manager $(R R H)$ :
"Do you think your practice has changed in any way as a result of the students being here?"

"Maybe hand hygiene, when they gave us the hand washing gel. They gave us books; we can consult from those books. We have about four textbooks on occupational therapy and mental health; it is helping us as we didn't have any at all"

This quote illustrates the value of these 'in-kind' contributions not as isolated donations but as part of active programs of intervention on the ground. The hand gel referred to by the respondent was manufactured as part of an ongoing THET-funded project on Infection Prevention Control. ${ }^{14}$

\section{Case Study 2: Reducing Maternal Mortality Through Infection Prevention Control}

The ground work for this intervention commenced several years earlier as part of the SVP project in response to concerns about the lack of Ebola preparedness in Uganda. In the first instance, an SVP volunteer began local production of alcohol hand rub in a public facility in Kampala. This worked extremely well. However, when the project funding stopped, so did production of the gel despite very minimal production costs and apparent high-level stakeholder support.

$\mathrm{K} 4 \mathrm{C}$ decided to repeat this process but with stronger sustainability elements built in including production of an accredited product (registered with the Uganda National Bureau of Standards) with potential for sales into the private sector (as a social enterprise). Having this on-going project in Kabarole provided exciting opportunities for student engagement in all aspects of the work. UK students working alongside local university (MMU) students have played an active role in all stages of this intervention including the Infrastructure Audit (a WHO instrument designed to assess the presence of sinks; running water, soap etc.); active hand hygiene training some of which was student-lead; the manufacture and marketing of the product and audit using a modified WHO Hand Hygiene Compliance Tool. This kind of managed intervention has quite a different impact to simply giving local staff bottles of hand gel.

${ }^{14}$ www.knowledge4change.org.uk/ 
One of the EEP students played a particularly important role in this project. He applied for a placement as a mature social work student. $\mathrm{He}$ had previously worked for over ten years in a pharmaceutical company (Apollo Scientific Research, Manchester) ${ }^{15}$ and began to apply his skills and knowledge in this area many months prior to deployment raising funding for infrastructural investments and working with his previous employer to provide over 2000 plastic bottles to help the hand gel production process. Invited to reflect on his impact on the ground he replied:

It is a case now of following it through because it is a long term project and I'm happy with that. I'm speaking to [local staff and UK Company] about it on a regular basis and I am happy to stay involved. Some things are worth putting energy into and this is one of them. I think seeds were sown with the hand gel.

Student placements have provided very valuable support to this intervention which has in return created a real-life experience of IPC interventions for the students. This student, who is now doing a doctorate at another university, is continuing to support the work in Uganda. His interview underlines the importance of project continuity to effective placements but also to local health systems. It also reminds us that many undergraduate students have extensive life experience and skills accrued prior to their current degree. Indeed, many of the EEP students had prior degrees and most of them have extensive work experience.

The Ugandan biomedical engineer who acted as the local supervisor of the prosthetic and orthotic EEP students commented on the value he and his University gained from the investments made in purchasing local materials for the EEP students to use in the manufacture of prosthetics for local patients. These also benefitted their Ugandan student peers and of course the patients they treated. At his request we also purchased a projector to help in the delivery of training during the placements and beyond:

We really benefited a lot because the school of orthopaedic medicine got materials that it had not gotten before and the school of orthopaedic technology also got materials plus the projector that went to the school of orthopaedic medicine. These things they needed but they could not get

${ }^{15}$ Apollo Scientific Research are now underwriting the costs of providing bottles and labels for the gel. 
them from the administration because of the limited funds so when this project came in we told them we should make sure that the money should support the actual school that is involved. It's not going to the entire institution and that really so much benefited us.

This case illustrates the contribution that carefully managed investments can make in a much-grounded manner - which top-down Aid has so patently failed to achieve. They are small things that are of immediate benefit to the placement students themselves who in this case presented their work every week using the projector. There are very many cases in Uganda of students taking vocational courses who lack the raw materials to undertake practical work. We have witnessed this through our sister construction project $(\mathrm{OBAAT})^{16}$ where students lack access to protective clothing (including boots) basic equipment and raw materials such as cement or sand to enable them to actually practice building work.

Once the partners begin to recognise the value of these contributions and the fact that no cash transfers will ever take place the project began to settle. Indeed, we are rarely now asked for cash unless students are placed in the larger hospitals where there is an existing culture of such payments from local as well as international institutions. Interestingly, even when local colleges are charged a fee for placements we are not asked to follow suit. We believe that this reflects two things: firstly, that the UK students are often more highly trained and able to contribute more immediately than their Ugandan peers (or at least those cohorts who come straight from school). And, secondly, linked to that, the presence of the professional volunteers who in effect supervise/mentor their own staff plus the Ugandan students are in effect making their own non-pecuniary contribution. It is not so much that we are passing the responsibility for student supervision onto already hard pressed Ugandan staff: rather that, as a project, we are contributing to multi-disciplinary training and mentoring clusters.

Context is all important and in the environment within which the students are placed in Uganda we believe this is an effective, ethical and sustainable way of organising student placements. Notwithstanding the care taken to prevent the cash nexus fostering corruption and jealousy, we have had several experiences of Ugandan health workers trying to

${ }^{16}$ http://www.vmminternational.org/one-brick-at-a-time-obaat/ 
exhort cash from students. In one case a student (who incidentally came herself from Kenya and was black) was immediately pressurised to take bribes from patients as the doctor clearly did on a daily basis. He advised her that she would not survive long in Kenya if she did not engage in this practice (assuming she was going to be working in Kenya). In this case, despite talking to the doctor concerned, we decided to move the student to another location. In another and more common case, staff asked students to purchase drugs ostensibly to treat sick patients. We are aware that whilst this may be legitimate in some cases, this is also a mechanism to fund drugs for sale or for use in their private practices. On that basis, the project has adopted a firm stance on all donations urging students to report any such requests and resist the temptation to donate even small items, such as sweets and toys as this in itself distorts the objectives of the placements and the wider project and generates unintended consequences.

\section{From 'Ninja Medicine’ to 'Neglected Processes'}

We talked above about the unintended consequences of service delivery and gap-filling. This is a major risk with professional volunteers and especially more senior cadres many of whom are attracted by the lure of 'ninja medicine'. ${ }^{17}$ Interestingly it has been less of a challenge with students perhaps because the students themselves respect the fact that they are primarily there to learn and more acutely aware of the risks of lone working. We have found this to be particularly the case with nursing, midwifery and allied health students who tend to show a higher level of humility, and perhaps a little less confidence (hubris) than medical students. The most pressing need in the Ugandan public health system is to attend to 'neglected processes' and get the basics right; if the basics could be improved we would be far less likely to see the kinds of emergencies that congest the main hospitals. EEP students played a very important role in supporting the back-to-basics orientation. In this capacity, they are supporting and working alongside local staff in critical role modelling

${ }^{17}$ This is a phrase used by a junior doctor in the SVP excited by the opportunities to save lives in emergency situations on a daily basis in the National Referral Hospital. 
positions and, in the process, relieving the burden on them as the following Ugandan midwife explains:

When a patient comes it is much quicker because everything is done on time when they are here. All the observations are done before we even touch the mother and the mothers do appreciate by the way. They come and tell us because they can't communicate with the students because of the language problem so they tell us. They say where did you get these doctors and nurses I wish you could keep bringing them because they are helping us a lot spending a lot of time on the mother so we wait less hours. Even in the late hours if we get a case they will come and help us. They ask questions when they don't understand because some of them are nurses and they also want to learn. For us here it is fantastic I may say.

[Does it make more work for you?]

No it is very easy it makes our work simpler. They were even very happy helping us with deliveries.

Involving students in preventive public health roles such as these can play a very important role in improving patient management, reducing congestion in overwhelmed referral hospitals and preventing damaging delays. Having said, that unless the students are placed in an environment of planned and active partnership founded on trust relationships these outcomes may not be realised. The respondent in the case above is a midwife in a health centre that we have been actively building links with for some years. Case Study 3 provides some background:

\section{Case Study 3: Sustainable Capacity-Building at Kagote Health Centre, Kabarole District}

The midwife in the case above is based at Kagote Health Centre III, a midwifery-led unit based in Kabarole District. The first project visit to Kagote Health Centre took place in May, 2014. At this time the facility had not delivered a mother for 16 years and the maternity unit was closed. In June $2014 \mathrm{~K} 4 \mathrm{C}$ organised a visit involving four self-funded students. Working alongside an SVP midwife volunteer and the wider project team the facility was opened for business within a month. We have continued to support this location through the deployment of professional volunteers and it became the first 'training site' for undergraduate placements. 
A combination of our Fair-Trade contributions, working in close partnership with OBAAT, resulted in the relocation of the laboratory, the construction of two patient waiting areas; the refurbishment and extension of the delivery and post-natal wards and the relocation and refurbishment of the Out Patients Department. We have also been awarded Commonwealth Professional Fellowships for three of the five midwives based in Kagote to undertake further midwifery training at Salford University. This Health Centre was recently pronounced by the District Health Officer to be a model for Kabarole District. We are now using this site as a training site placement for MMU midwifery students who are supervised by both the local midwives and the SVP volunteers. The benefits for patients of this intervention can be seen in the graph shown in Fig. 4.2 produced during an audit undertaken by a recent placement trainee (in this case a Graduate Trainee from the Central Manchester Foundation Trust):

The very positive experience referred to above, and echoed by all midwives we interviewed, was not always shared by other cadres and in other contexts where our engagement as a project was less well-established or supported by long-term volunteers. We noted above the problems of motivation in the Ugandan public health workforce. The impact of poor motivation and a local culture ${ }^{18}$ of absenteeism extends beyond the student's own learning to seriously reduce the potential impact of their presence. An on-going problem we face in placing students in Ugandan public health facilities is that we can only rarely rely on local staff to be present with any predictability or reliability. We are using the word 'culture' here to refer not in an essentialist way to Ugandan ethnicities but

${ }^{18} \mathrm{We}$ are using the word 'culture' here to refer not in an essentialist way to Ugandan ethnicities but rather to specific occupational sub-cultures within the public health sector. Corruption and absenteeism are a case in point. Interestingly we have found that absenteeism and poor time-keeping is less of a problem in midwifery in comparison to nursing, however, perhaps due to the fact that there is much greater pressure on midwives to be present on a 24/7 service. Many are not and many facilities operate a very limited week-day only service bit where this does take place mothers vote with their feet and either fail to access services at all or go directly to higher level referral facilities. On the whole midwives are far more likely to be present over the working day and to undertake night shifts than nursing staff working in Out Patient Departments who typically leave by $1 \mathrm{pm}$ to engage in other forms of employment and supplement subsistence wages. 




Fig. 4.2 Deliveries at Kagote health centre, Kabarole district. Source: Created by the authors based on data collected on site by an EEP Placement Trainee, working alongside local midwives

rather to specific occupational sub-cultures within the public health sector. Corruption and absenteeism are a case in point. Interestingly we have found that absenteeism and poor time-keeping is less of a problem in midwifery in comparison to nursing, however, perhaps due to the fact that there is much greater pressure on midwives to be present on a $24 / 7$ service. Many are not and many facilities operate a very limited week-day only service bit where this does take place mothers vote with their feet and either fail to access services at all or go directly to higher level referral facilities. On the whole midwives are far more likely to be present over the working day and to undertake night shifts than nursing staff working in Out Patient Departments who typically leave by $1 \mathrm{pm}$ to engage in other forms of employment and supplement subsistence wages.

At most health centres there is strong pressure on nursing staff to 'clear the lines' (of patients) before $1 \mathrm{pm}$. This encourages staff to rush and is a 
major factor explaining the lack of effective patient monitoring and triage. In this environment, local staff may find having students around doing monitoring and triage effectively actually slows them down and this can generate some resentment to the presence of both students and professional volunteers. Patients are fully aware of this culture. As a result, many facilities (and potential placement locations) empty after $1 \mathrm{pm}$. This situation is reflected in the following responses:

She only worked from 9 until 1 and then she'd go home. To begin with I would stay until one and then she sends me home. (Occupational Therapist, Uganda)

I think they're just lazy because it always finishes by lunchtime: they can arrive late, see their patients quickly and then go home for lunch and then not turn up for the rest of the day. (Podiatrist, Uganda)

Where projects are actively engaged on the ground and where professional volunteers are in place this is much easier to predict and manage but nevertheless remains a major challenge for placement managers. An obvious response to this tension for placement managers is to place students in private and mission (not-for profit) facilities where occupational cultures (and human resource management) is quite different and supports a full working day and potentially more effective supervision. Our (ethical) commitment to health systems change could not support this approach and students would gain very little exposure to the challenges facing health systems in low resource settings if we took the easy option. Our commitment to co-presence further exacerbates this problem and the challenge of achieving active clinical engagement across the working day. If we allowed professional volunteers to engage in service delivery roles in the absence of local staff we could provide the supervision necessary to enable students to engage in clinical work. We have witnessed this type of situation on many occasions during visits to health centres. It is not at all unusual to see a quite junior overseas volunteer working with a small group of foreign students (often from different countries) on their own in a health facility. We would not regard this type of activity as either safe or ethically acceptable encouraging as it does systems damaging practices of absenteeism and moonlighting (Ackers et al. 2016).

The student in the following case explains how this situation affected his placement and the impacts associated with it. He experienced absenteeism in the hospital but also when the students had 
agreed to organise First Aid training for local ambulance staff and MMU students. Fortunately, he reflected on this as part of his own learning:

There was a lot of absenteeism from Ugandan staff - some days I'd be going to the operating theatres and you would be all ready to go but no staff there so theatre would be cancelled. This obviously affects your entire day. With the staff absenteeism, that could affect our time because we could have prepared way in advance to go to do something and people don't turn up and then it is cancelled so that can be frustrating. It appears that even if they didn't come to work, they would still get paid, and then they questioned why there was such an issue with attendance. It's more about the situation in Uganda as opposed to us, but I don't know how we could possibly get it changed as it is outside our control. It was just interesting to see. (Adult Nurse, Uganda)

In another case the student suggested that local staff tried to 'put them out of their way'; they also observed that when the UK obstetrician volunteer was around local staff took the opportunity not to come to work (something we try hard to prevent):

I think they just wanted us out of the way, so they put us in a room triaging, but they didn't listen to anything we said. When [professional volunteer] came the doctors stopped turning up because she was there to do the work. (Adult Nurse, Uganda)

In another context, students noted that when the Project Managers were around staff behaved quite differently towards them:

It was really laid back. Have you heard that if it's raining people don't go to work they just stay indoors? The impression that we got is that when yourself and $[\mathrm{X}]$ go over, it's like the CQC (Care Quality Commission) coming to the hospitals over here, so there were certain staff that weren't very friendly towards us, or motivated. But when [X] was here she was saying that they were absolutely lovely they did everything but we had seen a completely different side of that. (Nurse, Uganda)

As a project, we learnt that placing some cadres of students presented more challenges than others and mental health was a case in point. In the 
first instance, we placed these students in the Mental Health Unit at the regional referral hospital aware that there was no community mental health in the Ugandan public system. This placement proved a serious challenge as the demands on language were much higher (most patients not speaking English and language being a much more important component of diagnosis and treatment). The Unit also managed the most serious cases with very few staff. This facility also completed its work around lunchtime each day and our interview with a local health worker expressed concerns at the lack of opportunity for active student engagement and the pressure this put on her:

There are days here where I don't know what to do with these students when I am not busy and you have to make them busy so that was a very big challenge. (Health Worker, Uganda)

In this and other similar situations the potential for effective knowledge mobilisation and systems impact was limited. This did not imply that knowledge was being exchanged but the resource and cultural environment restricted its benefit:

Response: "They shared knowledge especially when we asked them how they do it in their country. Comparing approaches and how they handle things".

Interviewer: "Do you think that knowledge could be applied here?"

Response: "Not really, maybe. What is practical we can't do here. Here the patient comes and what we normally do is say what we think is right for them; which is wrong. Here patients don't even know their rights, they don't know what they are supposed to expect. There (in the UK) they say the patient has to be told all their options and they make a choice and if they don't want it that is ok; maybe that is a difference I saw. Here it may not be very practical for us because our patients don't even know what they are suffering from when they come. They don't know where to get information from so if we leave them to decide they may not do it; that is a limitation. The doctor will decide which medicine to give them and they take it; we convince them to take it but they really don't know what they are taking and sometimes they don't know the side effects but the patient should surely know before taking a drug they should expect the side effects."

(Nurse, Uganda) 
As this was a new placement group and we did not have long-term volunteers in this field we monitored this carefully and subsequently placed mental-health students with a mental-health specialist who runs a local NGO. We have noted above our reasons for not placing students outside of the public-health system. In some situations, where services are barely in existence or professions not yet developed this was the only option and the NGOs involved were actively interfacing with local public health facilities. This is certainly the case with the Youth and Women Foundation (YAWE).

\section{Case Study 4: The Youth and Women Foundation (YAWE) Mobile Clinic}

For the reasons described above, K4C has partnered with the Youth and Women Foundation (YAWE). ${ }^{19}$ YAWE focus on youth and gender empowerment with a particular emphasis on 'living positively' (with HIV). The Director of YAWE is a mental-health specialist. YAWE had been donated a mobile clinic to undertake community outreach work in rural villages and refugee camps. When we visited YAWE to discuss potential placements for mental-health nurses we found that the vehicle was not being used due to a lack of resource (fuel and manpower). We felt that the mobile clinic would offer unique opportunities for students and also critical complementary services in hard-to-reach areas. On that basis, we agreed to fund the costs of using the vehicle once or twice a month. During the outreach visits, local staff and the EEP students work alongside village health teams who mobilise the local community providing cervical screening, family planning, HIV diagnosis, care and treatment and diabetes screening alongside other public health services. The Director describes the value of these visits to his project and the local community:

They do home visits - nursing care, malaria - wounds - those who need medication and also our mobile clinic they join the team when we go to the field - we provide fully fledged healthcare services including screening for HIV, diabetes because most people in the villages don't get the chance to get diagnoses because the equipment isn't there. The students from your program are very good - they do that - we provide them with strips - they

${ }^{19}$ www.yawefoundation.webs.com 
also check BPs and cervical cancer screening but most of them have learnt that here because they come when they are not so skilled but they participate. They participate in the actual procedures.

When the team go out one of the professional volunteers usually accompanies them. The outreach clinics have proved particular attractive to placement students. As project managers, we were concerned that placing mental-health students at YAWE may put a burden on local staff. Whilst the supervisor admitted that hosting the students does imply an investment on his part he said he enjoys this and recognises the wider reciprocity involved:

Interviewer: "I was just trying to work out if you are making special plans
distracting you from your normal work - are we making extra
work for you?"
"It is true sometimes I feel they need attention especially the
Respontal health students so I feel they benefit from having a person
like me (a mental health specialist) with them so this week I have
set up a program for them".
Interviewer: "So you are doing extra work for the students?"
Response: "Yes but they had a week of supervision and I wanted the students
to see how mental health counsellors here work and what tools they
use - bow they diagnose here. Your project supports our outreaches
(mobile clinic) so we are happy to help. I enjoy working with
students and so long as it is planned it is OK".

The final case study presented below is included as an example of project development and how students (in this case NHS Management Trainees) can be involved in quite strategic planning roles focused on some of the most serious obstacles to health systems change. This also illustrates the potential for multi-disciplinary integrated interventions engaging the kinds of knowledge clusters we referred to earlier on.

\section{Case Study 5: Building the Case for Public-Private- Partnerships in Kabarole District}

As noted in Chapter 2, the EEP project was designed to provide placements for students. Most of the EEP participants have been studying at undergraduate level although many of them will already hold first degrees. Some of the students were also studying at Masters Level and several were involved in 
data collection for their doctorates. ${ }^{20}$ Towards the end of the Health Education England project we were presented with an opportunity to organise a placement for NHS Management Trainees. These trainees are all graduates undertaking a fast-track management training with a view to becoming managers in the UK NHS. As a project, we were at the point of making a critical decision of whether to embark on a new project to restore functionality to a Health Centre IV facility that was failing to deliver effective maternity services.

Given our knowledge of this process and the very serious concerns we had about trying to get emergency obstetric services going in the absence of a doctor and our experience of trying to mobilise Ugandan doctors in these environments (Ackers et al. 2016) we mooted the idea of a PublicPrivate-Partnership drawing on a model explicitly advocated in the Ministry of Health Strategic Plan (2012). The success of this intervention, in our experience, rested on the ability to devolve the budget and responsibility for human and physical resource management to a PPP Management team. This represented an ambitious and entirely untested idea. The four NHS Management Trainees from the Central Manchester Foundation Trust were tasked to work alongside one of the project managers spending a month in Uganda to conduct a thorough scoping analysis and audit and prepare a proposal for a PPP intervention. The Trainees conducted some very high-quality work engaging in active negotiation and audit-related activity combined with interactions with local health workers to create the first steps in this potentially exciting new venture. $^{21}$

\section{Relationship and Capacity-building in Partner UNIVERSITIES}

The EEP model involves another element of capacity-building that has been of great value to the local University and health system. The professional volunteers funded through the EEPs each co-teach one module on a new BSc Midwifery programme at Mountains of the Moon University (MMU). The programme was planned with considerable support from $\mathrm{K} 4 \mathrm{C}$ and

${ }^{20}$ These students received funding from Santander.

${ }^{21}$ The outcome of this venture will not be clear for some time. 
three professional volunteers and welcomed its first cohort of students in August 2015. It would have proved very difficult to get this programme off the ground in Uganda in the absence of professional volunteers as there are so few people qualified to teach the programme at degree level. ${ }^{22}$ This model is focused on sustainability and capacity-building and co-presence in the teaching environment has been insisted upon in order to ensure that institutional memory and resources are built up for the future. The programme has also involved the active encouragement of MMU and diploma level midwives in selected local facilities to come to the UK to undertake recognised training programmes at Masters Level in order to build capacity in Uganda but also support mutual understanding of educational programmes and training systems. We have planned the whole process to focus on a small number of health centres which we are developing as established training sites both for UK and Ugandan students on placement (including Kagote). Whilst ensuring optimal learning outcomes for UK students this process has ensured that Ugandan students can begin to be supervised in fully functioning health facilities.

In some cases, the Ugandan fellows have been trained in research methods (rather than clinical areas) in order to build research capacity at MMU but also invest in the evaluation potential of our Ugandan colleagues. This will enable us in future to engage in genuine coresearch activity. Asked to reflect on the contribution of the student placements to this area of activity the academic lead of Health Sciences responded;

[Is there anything we can do to improve to improve impact?]

I've been very impressed with the quality of the students' teaching of course they were mature students - but they did a really beautiful job teaching our students and our students really appreciate someone coming in and teaching them from outside (even if they are also students) they are teaching something appropriate to our student's environment and something practical too. I think it would be good to keep that in mind and plan ahead and make sure topics are needed to be covered - if

${ }^{22}$ Uganda faced a 'catch22' situation as the rules required that a degree programme in midwifery is taught by staff with a degree in midwifery. At the present time the number of midwives in Uganda with a degree can be counted on one hand. 
we can think about it and plan ahead and the students are interested in teaching and it is good for them.

\section{Conclusions}

The results of the EEP evaluation suggest that undergraduate placements can form the basis of valuable capacity-building work in low resource settings. We have been somewhat surprised at these results building as they do on several years' evaluation of the impact of far more experienced volunteers and the demand from host settings for the most senior and experienced professionals. Reflecting upon this apparent contradiction and the sense that student placements when organised in structured and deeply grounded 'knowledge clusters' may facilitate a higher level of sustainable systems change than the isolated deployment of highly qualified volunteers the logic is clear. The human resource environment in Uganda is such that for most of the time there are no doctors in situ and facilities are run by nurses, midwives and clinical/anaesthetic officers. The majority of staff in many facilities will be students themselves often at an early stage in their training with little concrete knowledge or experience and typically no effective supervision.

Referral systems are structurally damaged in the overwhelming majority of lower-level health facilities and basic neglected processes (patient monitoring; record keeping; audit; infection prevention control; and prescribing behaviour) are rarely in place. In these circumstances, we may question the merits of deploying highly qualified physicians to Uganda as they will rarely work in sustained co-present relationships with their peers. Deploying cadres of staff such as midwives and nurses facilities a more immediate engagement and the formation of highly effective training clusters supporting bi-lateral mentoring and knowledge exchange. This, coupled with the ability to identify and remove minor 'snagging' problems and leverage some conditionality within the local health system wherever possible through the fair-trade premium arguably constitutes the most efficient and effective intervention whilst also reducing the system destabilising effects associated with labour substitution UK students are far less likely to create the kinds of 'malignancy' referred to by Moyo (2009). The overall impact of EEPs is dampened by systems features and, most notably, staff absence and poor time-keeping. If staff are not presents and systems effectively 
close down in the afternoons then opportunities for knowledge mobilisation close down too. Responsibility here rests with the Uganda authorities to manage their human resource more effectively to improve motivation and reduce the need for moonlighting. An easy solution for placement providers would be to place students in better managed and remunerated private and mission facilities. As a project we do not believe that this 'solution' complies with ethical principles or gives the UK students the best understanding of global health.

Open Access This chapter is licensed under the terms of the Creative Commons Attribution 4.0 International License (http://creativecommons.org/licenses/ by $/ 4.0 /)$, which permits use, sharing, adaptation, distribution and reproduction in any medium or format, as long as you give appropriate credit to the original author(s) and the source, provide a link to the Creative Commons license and indicate if changes were made.

The images or other third party material in this chapter are included in the book's Creative Commons license, unless indicated otherwise in a credit line to the material. If material is not included in the book's Creative Commons license and your intended use is not permitted by statutory regulation or exceeds the permitted use, you will need to obtain permission directly from the copyright holder.

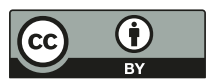

